\title{
Metacarpophalangeal Joint 3
}

National Cancer Institute

\section{Source}

National Cancer Institute. Metacarpophalangeal Joint 3. NCI Thesaurus. Code C102318.

A condyloid synovial joint within the third digit of the hand connecting the metacarpal to the proximal phalanx. 